\title{
Bancos de Sementes de Plantas Daninhas em Cultivos de Mandioca na Amazônia Central ${ }^{1}$
}

\author{
Weed Seed Banks in Cassava Cultivations in Central Amazon \\ COSTA, J.R. ${ }^{2}$, MITJA, D. ${ }^{3}$ e FONTES, J.R.A. ${ }^{4}$
}

\begin{abstract}
RESUMO - Este estudo investigou a densidade de sementes e a riqueza de espécies dos bancos de sementes de plantas daninhas em cultivos de mandioca (Manihot esculenta), em pequenas propriedades rurais localizadas em Manacapuru, Amazonas. Nos cultivos foram estabelecidas quatro parcelas, de onde foram coletadas 20 amostras $\left(0,0225 \mathrm{~m}^{2}\right)$ de $15 \times 15 \mathrm{~cm}$ $\left(0,0225 \mathrm{~m}^{2}\right)$ na camada de $0-5 \mathrm{~cm}$ e três amostras nas profundidades de $5-10$ e $10-30 \mathrm{~cm}$, totalizando 104 amostras. Para a contagem e identificação das sementes, utilizou-se a técnica de emergência de plântulas em casa de vegetação. As plântulas foram identificadas, inicialmente, por morfotipo e, quando possivel, até espécie. O monitoramento foi feito durante nove meses. Houve diferença significativa na densidade de sementes (Kruskal Wallis, 5\%; $\mathrm{p}<0,05)$ entre as parcelas e verificou-se uma média de 5.113 sementes $\mathrm{m}^{-2}$, na profundidade de $0-5 \mathrm{~cm}$. Na profundidade de $5-10 \mathrm{~cm}$, os cultivos de mandioca apresentaram uma média de $1.111,25$ sementes $\mathrm{m}^{-2}$, enquanto na profundidade de $10-30 \mathrm{~cm}$ a média foi de 285 sementes $\mathrm{m}^{-2}$. Os bancos de sementes dos cultivos de mandioca foram constituidos principalmente por espécies herbáceas - características de áreas agrícolas e ambientes perturbados. A realização de uma capina anual não foi suficiente para controlar as plantas daninhas e reduzir a intensidade da interferência negativa sobre a cultura, sendo necessários maiores cuidados nos períodos que antecedem as épocas de floração e produção de sementes, de acordo com a disponibilidade de mão de obra.
\end{abstract}

Palavras-chave: sementes no solo, herbáceas, manejo, Manihot esculenta.

ABSTRACT - This study aimed to evaluate seed density and number of species in a weed seed bank collected from four different cassava family farms. The farms were located on highway AM-07O in the state of Amazonas, Brazil. Four plots were established in each cassava crop, from which $2015 \times 15 \mathrm{~cm}$ samples. $\left(0.0225 \mathrm{~m}^{2}\right)$ at $0-5 \mathrm{~cm}$ depth level and three samples at 5-10 and 10-30 depth levels were collected, totalizing 104 samples. For seed counting and identification, plantlet emergence technique under greenhouse conditions was used. The plantlets were initially identified by morphotype and when possible, even by species. Monitoring was conducted during 9 months. A significant difference in seed density was found among the plots (Kruskal Wallis, 5\%; $p<0.05)$ and an average of 5.113 seeds $\mathrm{m}^{-2}$ at $0-5 \mathrm{~cm}$ depth. At the depth of $5-10 \mathrm{~cm}$, the cassava crops presented an average of 1.111 seeds $\mathrm{m}^{-2}$, while at the $10-30 \mathrm{~cm}$ depth, the average was 285 seeds $m^{-2}$. The seed banks of the cassava crops were basically herbaceous species typical of agricultural lands and disturbed environments. Weed cut once a year was not sufficient to control weed plants and reduce negative weed interference intensity. Thus, therefore, greater care is necessary during the periods before flowering and seed production, according to labor availability.

Keywords: seeds in soil, herbaceous species, crop management, Manihot esculenta.

Recebido para publicação em 26.6.2008 e na forma revisada em 13.11.2009.

2 M.Sc., Pesquisadora da Embrapa Amazônia Ocidental, <joanne.regis@cpaa.embrapa.br>; Fone: (92) 3303-7929 - Fax: 3303-7820, Rodovia AM-10, Km 29, Caixa Postal 319, 69010-970 Manaus-AM; ${ }^{2}$ Dra., Pesquisadora do IRD/Embrapa Cerrados, <mitja@cpac.embrapa.br>, Fone: (61) 3388-9898 - Fax: (92) 3388-9879, Rodovia BR 020 Km 18, Caixa Postal: 08223, 73310-970 Planaltina-DF; ${ }^{3}$ Dr., Pesquisador da Embrapa Amazônia Ocidental, <zeroberto.fontes@cpaa.embrapa.br>.

Planta Daninha, Viçosa-MG, v. 27, n. 4, p. 665-671, 2009 


\section{INTRODUÇÃO}

A infestação de plantas daninhas em áreas agrícolas exerce influência negativa sobre a produtividade e o consequente abandono da terra pouco tempo após a instalação dos cultivos. Os prejuízos na produção ocorrem devido à interferência negativa sobre as plantas cultivadas e ao efeito da competição e da alelopatia (Sousa, 1995). A competição das plantas daninhas com a cultura da mandioca nos primeiros três meses reduz sensivelmente o rendimento desta, verificando-se que, nesse período, ocorre maior concorrência pelos fatores necessários ao desenvolvimento das plantas (Pacheco et al., 1974).

Em geral, os efeitos negativos das plantas daninhas em culturas agrícolas decorrem tanto do aumento da sua densidade quanto da duração de sua interferência. Dessa forma, o conhecimento da dinâmica dos bancos de sementes é fundamental para a definição de práticas de manejo apropriadas, que venham a reduzir as perdas de rendimento associadas (Fernandes-Quintanilla, 1988; Vismara et al., 2007). Os bancos de sementes caracterizamse como o estoque de sementes acumulado no solo (Cavers, 1995) e representam papel ecológico importante no suprimento de novos indivíduos para as comunidades vegetais ao longo do tempo (Carmona, 1992). Com o banco de sementes é possivel a formação de um estoque de material genético, que será prontamente estimulado para seu desenvolvimento quando do aparecimento das condições propícias. A natureza e a intensidade do distúrbio em uma área determinam a resposta do banco de sementes a ele. A severidade do fogo, por exemplo, tem mostrado forte influência sobre o banco de sementes (McGee \& Feller, 1993).

O conhecimento das características dos bancos de sementes, como número de espécies, quantidade de sementes e distribuição destas no perfil do solo, permite projeções sobre a velocidade e composição florística dos estádios iniciais da regeneração e pode fornecer uma estimativa do potencial da regeneração após o distúrbio (Thompson \& Grime, 1979; Garwood, 1989).

Estudos básicos sobre biologia de plantas daninhas e, em especial, daquelas que infestam áreas tropicais e subtropicais são escassos
(Canossa et al., 2007). Estimativas do potencial de sementes em áreas agrícolas são de extrema importância na previsão de infestações de plantas daninhas, no melhor conhecimento da dinâmica das espécies em distintas situações e, consequentemente, na proposição de programas mais racionais de manejo em cada situação (Carmona, 1995).

O objetivo deste trabalho foi estimar o banco de sementes de espécies daninhas em áreas cultivadas com mandioca (Manihot esculenta), em pequenas propriedades agrícolas localizadas na zona rural de Manacapuru, Estado do Amazonas, Brasil.

\section{MATERIAL E MÉTODOS}

A área de estudo localiza-se no município de Manacapuru (Amazonas), entre o rio Solimões e a rodovia AM 070 (Manaus Manacapuru), a $3^{\circ} 16^{\prime} 20^{\prime \prime}$ de latitude sul e $60^{\circ} 33^{\prime} 07^{\prime \prime}$ de longitude oeste. Nesse local existem dois ramais, onde, em 1986, foram assentados agricultores familiares. Uma parcela está localizada no ramal Nova Esperança, que liga o $\mathrm{km} 64$ da rodovia AM 070 ao lago do Calado, o qual desemboca no rio Solimões. As outras parcelas estão localizadas em lotes no ramal do Laranjal, que liga o $\mathrm{km} 62$ da rodovia AM 070 ao rio Solimões.

Em um transecto com $50 \mathrm{~m}$ de comprimento localizado na faixa central de oito parcelas (quatro cultivos de mandioca e quatro sistemas agroflorestais), foram coletadas 20 amostras de solo na profundidade de $0-5 \mathrm{~cm}$, num total de 104 amostras. Foi utilizado um gabarito com $15 \times 15 \mathrm{~cm}$ e $5 \mathrm{~cm}$ de profundidade para a coleta do banco de sementes. Foram coletadas, ainda, seis amostras dos bancos de sementes no perfil do solo, sendo três na profundidade de 5 a $10 \mathrm{~cm}$ e três na profundidade de 10 a $30 \mathrm{~cm}$, somando 48 amostras.

A casa de vegetação foi inteiramente cercada com sombrite $60 \%$ e coberta por telhas transparentes. As amostras de solo foram colocadas em bacias redondas, com diâmetro de $20 \mathrm{~cm}$ e profundidade de $8 \mathrm{~cm}$. As bacias foram furadas para permitir a drenagem. A liteira coletada foi lavada para a retirada de sementes, e a água utilizada foi colocada em sua respectiva bacia. As amostras ficaram sob temperatura ambiente e sujeitas aos 
fotoperíodos naturais. As irrigações foram feitas conforme a necessidade do momento, a fim de manter o solo sempre úmido.

As contagens e identificações das plântulas foram feitas em casa de vegetação, em intervalos que variaram entre 15 e 35 dias, conforme o fluxo de emergência. No primeiro mês, a contagem e a identificação foram feitas quinzenalmente, e esse intervalo foi prolongado de acordo com a diminuição do fluxo. $\mathrm{O}$ monitoramento teve a duração de nove meses, com 11 levantamentos.

Em principio, as plântulas foram separadas por morfotipos - um exemplar visualmente reconhecivel. Quando possivel, a identificação chegou à taxa de espécie. Houve espécies que apresentaram poucos indivíduos e não sobreviveram até que fosse possivel sua identificação. A identificação das plântulas foi feita por comparação com exsicatas encontradas nas coleções do herbário do Instituto Nacional de Pesquisas da Amazônia (INPA), do laboratório da ORSTOM - INPA e do Museu Emílio Goeldi, em Belém (Pará). Contudo, a inexistência de coleções de referência de material botânico em estado juvenil, associada às dificuldades de identificação de indivíduos nessa fase, foram fatores que limitaram a elaboração de uma listagem completa das espécies que compõem o banco de sementes. Nove espécies ficaram sem identificação.

Os dados foram analisados utilizando testes não paramétricos, uma vez que não apresentaram uma distribuição normal. Os valores médios de densidade de sementes, nas profundidades de $0-5 \mathrm{~cm}$, foram comparados pelo teste de Kruskal-Wallis a $5 \%$ de probabilidade. O Índice de Similaridade de Jaccard (ISJ) foi usado para estimar o grau de semelhança florística entre as parcelas estudadas (MullerDombois \& Ellenberg, 1974):

$$
\operatorname{ISJ}(\mathrm{A}, \mathrm{B})=\mathrm{C} /(\mathrm{A}+\mathrm{B}-\mathrm{C}) \times 100
$$

em que ISJ $(A, B)=$ Índice de Similaridade de Jaccard entre as áreas A e $B ; C=n^{\circ}$ de espécies comuns nas áreas $A$ e $B ; A=n^{\circ}$ de espécies da comunidade $A ;$ e $B=n^{\circ}$ de espécies da comunidade $\mathrm{B}$.

\section{RESULTADOS E DISCUSSÃO}

\section{Densidade de sementes}

Na profundidade de $0-5 \mathrm{~cm}$, foram contadas 9.204 sementes nos quatro cultivos de mandioca, o que correspondeu, em média, a 5.113 sementes $\mathrm{m}^{-2}$ (Tabela 1 ). Verificou-se diferença significativa (Kruskal Wallis, $\mathrm{p}<0,001)$ entre as densidades de sementes das quatro parcelas de mandioca, cujas médias apresentaram grande amplitude entre si, variando de 44 a 187.

A distribuição das sementes no perfil do solo indicou decréscimo no número de espécies e indivíduos de plantas daninhas com o aumento da profundidade. Foi observado decréscimo no número de sementes a partir da profundidade de $0-5 \mathrm{~cm}$, com média de $1.111,25$ sementes $\mathrm{m}^{-2}$ na profundidade de 5$10 \mathrm{~cm}$ e 285 sementes $\mathrm{m}^{-2}$ na de $10-30 \mathrm{~cm}$, respectivamente.

\section{Riqueza de espécies}

Foram encontrados 52 morfotipos e espécies, sendo 34 morfotipos identificados até espécie $(65,38 \%), 9$ até gênero $(17,31 \%), 5$ até familia botânica $(9,62 \%)$ e 4 permaneceram como morfotipos $(7,69 \%)$.

O indice de Jaccard mostrou que há baixa similaridade na composição florística das parcelas de mandioca (Tabela 2).

Tabela 1 - Médias, desvios e erros-padrão das densidades de sementes germinadas a partir dos bancos em cada cultivo de mandioca $(\mathrm{N}=20)$, na profundidade de $0-5 \mathrm{~cm}$

\begin{tabular}{|c|c|c|c|c|c|}
\hline Cultivo & $\begin{array}{c}\text { № de sementes } \\
\text { encontradas }\end{array}$ & № de sementes $\mathrm{m}^{-2}$ & Média por parcela & Desvio-padrão & Erro-padrão \\
\hline MAN1 & 3.362 & 7.471 & 168 & 203,22 & 45,44 \\
\hline MAN2 & 3.748 & 8.329 & 187 & 122,35 & 27,36 \\
\hline MAN3 & 1.211 & 2.691 & 61 & 115,95 & 25,93 \\
\hline MAN4 & 883 & 1.962 & 44 & 26,57 & 5,94 \\
\hline
\end{tabular}


Tabela 2 - Índice de similaridade de Jaccard (\%) nas parcelas de mandioca. Manacapuru-AM

\begin{tabular}{|l|c|c|c|c|}
\hline Cultivo & MAN3 & MAN1 & MAN4 & MAN2 \\
\hline MAN3 & & 30 & 46 & 38 \\
\hline MAN1 & & & 52 & 29 \\
\hline MAN4 & & & & 47 \\
\hline
\end{tabular}

As principais espécies encontradas nos cultivos de mandiocas foram de porte herbáceo e consideradas espécies pioneiras, características de áreas agrícolas e ambientes perturbados (Tabela 3). As diferentes coberturas de plantas nas áreas estudadas podem ter influenciado os resultados encontrados, pois a composição de sementes oriundas da chuva varia em função da proximidade da mata e das zonas agropecuárias (Gorchov et al., 1993). Segundo Canossa et al. (2007), a presença de cobertura na superfície do solo tem implicação direta na emergência de muitas espécies de plantas daninhas, como no caso de coberturas mortas, as quais podem apresentar efeito estimulador ou inibidor na germinação das sementes e emergência de plântulas, dependendo da espécie que faz a cobertura e da biomassa por ela produzida (Vidal \& Trezzi, 2004; Canossa et al., 2007). Para Correia (2005), resíduos vegetais mantidos na superfície do solo alteram a umidade, luminosidade e temperatura do solo - principais fatores que influenciam a germinação de sementes e a emergência de plântulas. Além disso, o processo de decomposição da cobertura morta na superfície do solo libera uma série de compostos orgânicos que podem interferir na germinação e emergência de plantas daninhas (Souza et al., 2006). Outro fator que pode ter contribuído para os resultados encontrados é o número de queimadas. Foram feitas de duas a três queimas como forma de limpeza das áreas com cultivos de mandioca. A queima influencia os bancos de sementes no solo, provocando a morte das sementes ou sua germinação. $\mathrm{O}$ fogo pode tanto estimular quanto destruir o banco de sementes, mesmo em ecossistemas cujas espécies evoluíram tendo o fogo como fator de seleção (McGee \& Feller 1993; Garcia-Nuñes et al., 2003). Temperaturas elevadas provocam estresse, ocasionando, então, inibição ou dormência térmica, ou até mesmo perda de viabilidade. Temperaturas elevadas acarretam uma diminuição do suprimento de aminoácidos livres, da síntese proteica, da sintese de RNA e das reações anabólicas. De maneira geral, altas temperaturas desnaturam proteínas, alteram a permeabilidade da membrana e ocasionam perda de material, enquanto as baixas retardam as taxas metabólicas até o ponto de inibição da germinação (Castro \& Vieira, 2001).

Outros fatores importantes que afetam as sementes no solo são as condições ambientais, como os locais favoráveis para a germinação (Epp, 1987). Em ambientes mais abertos, como os cultivos de mandioca, há mais exposição da superfície 1 do solo à luz solar, com aumento de temperatura, o que estimula a germinação e a diminuição do estoque de sementes no solo.

O preparo do solo, o controle de plantas daninhas realizado com enxada e a colheita das raízes da mandioca provocam constantes perturbações no solo e, nessas condições, as sementes dormentes podem germinar devido à quebra da dormência provocada pela exposição à luz solar, com o revolvimento do solo (Parker et al., 1989). Esse revolvimento pode contribuir tanto para o enterramento de sementes em maiores profundidades quanto para o posicionamento daquelas antes enterradas próximas à superfície. Noldin et al. (2006) verificaram que a longevidade de sementes de arroz-vermelho (Oryza sativa) enterradas em camada de solo mais superficial (até $5 \mathrm{~cm}$ ) foi muito reduzida quando comparada à daquelas enterradas em maior profundidade (12 a $25 \mathrm{~cm}$ ). Quando as sementes não dormentes retornam à superficie, provavelmente germinam, mas as dormentes poderão retornar para uma profundidade maior, pelo cultivo posterior (Cavers \& Benoit, 1989).

A ocorrência de espécies de plantas daninhas de porte herbáceo nas áreas avaliadas pode ter garantido a presença de um grande número de sementes no solo, pois elas são capazes de produzir grande quantidade de sementes capazes de permanecer viáveis por longos períodos.

A ausência de espécies típicas da floresta tropical primária neste estudo, pelo menos entre as espécies identificadas, pode estar relacionada à ausência desses indivíduos em fase de frutificação na área estudada ou em 
Tabela 3 - Lista dos morfotipos e espécies encontrados nos cultivos de mandioca, nas três profundidades estudadas, com as respectivas famílias e hábitos de vida

\begin{tabular}{|c|c|c|}
\hline Nome da espécie & Família & Hábito de vida \\
\hline Borreria latifolia & Rubiaceae & $\mathrm{H}$ \\
\hline Cecropia spp. & Cecropiaceae & $\mathrm{L}$ \\
\hline Chromolaena odorata & Asteraceae & $\mathrm{H}$ \\
\hline Conyza bonariensis & Asteraceae & $\mathrm{H}$ \\
\hline Croton trinitatis & Euphorbiaceae & $\mathrm{H}$ \\
\hline Cyathula pilosa & Amaranthaceae & $\mathrm{H}$ \\
\hline Dichromena ciliata & Cyperaceae & $\mathrm{H}$ \\
\hline Emilia florsbergii & Asteraceae & $\mathrm{H}$ \\
\hline Erechtites hieracifolia & Asteraceae & $\mathrm{H}$ \\
\hline Fimbristyllis annua & Cyperaceae & $\mathrm{H}$ \\
\hline Irlbachia alata & Gentianaceae & $\mathrm{H}$ \\
\hline Lindernia crustacea & Scrophulariaceae & $\mathrm{H}$ \\
\hline Lindernia diffusa & Scrophulariaceae & $\mathrm{H}$ \\
\hline Ludwigia leptocarpa & Onagraceae & $\mathrm{H}$ \\
\hline Marsyfrianthes chamaedrys & Lamiaceae & $\mathrm{H}$ \\
\hline Melochia melessaefolia & Sterculiaceae & $\mathrm{H}$ \\
\hline Mikania cordifolia & Asteraceae & $\mathrm{CH}$ \\
\hline Mimosa spruceana & Mimosaceae & $\mathrm{CL}$ \\
\hline Mollugo verticillata & Aizoaceae & $\mathrm{H}$ \\
\hline Oxalis cytisoides & Oxalidaceae & $\mathrm{H}$ \\
\hline Ophioglossum sp. & Ophioglossaceae & $\mathrm{H}$ \\
\hline Panicum laxum & Poaceae & $\mathrm{H}$ \\
\hline Paspalum decumbens & Poaceae & $\mathrm{H}$ \\
\hline Paspalum multicaule & Poaceae & $\mathrm{H}$ \\
\hline Phyllanthus niruri & Euphorbiaceae & $\mathrm{H}$ \\
\hline Phyllanthus orbiculatus & Euphorbiaceae & $\mathrm{H}$ \\
\hline Phyllanthus urinaria & Euphorbiaceae & $\mathrm{H}$ \\
\hline Potomorphe peltata & Piperaceae & $\mathrm{H}$ \\
\hline Sabicea áspera & Rubiaceae & $\mathrm{CH}$ \\
\hline Scoparia dulcis & Scrophulariaceae & $\mathrm{H}$ \\
\hline Solanum rugosum & Solanaceae & SL \\
\hline Solanum sp. & Solanaceae & SL \\
\hline Solanum sp. & Solanaceae & SL \\
\hline Solanum sp. & Solanaceae & SL \\
\hline Sp61 (Aciotis circacifolia e Miconia sp.) & Melastomataceae & $\mathrm{H}$ \\
\hline Sp5 (Cyperus compressus e Rhynchospora nervosa) & Cyperaceae & $\mathrm{H}$ \\
\hline Trema micrantha & Ulmaceae & $\mathrm{L}$ \\
\hline Vernonia remotiflora & Asteraceae & $\mathrm{H}$ \\
\hline Vernonia sp. & Asteraceae & $\mathrm{H}$ \\
\hline Vismia sp. & Clusiaceae & $\mathrm{L}$ \\
\hline Vismia sp. & Clusiaceae & $\mathrm{L}$ \\
\hline
\end{tabular}

SL: sublenhosa; L: lenhosa; H: herbácea; CL: cipó Lenhoso; CH: cipó herbáceo; HNI: hábito não identificado.

suas proximidades (Hopkins \& Graham, 1983). Suas sementes apresentam curta viabilidade e incapacidade de entrar em dormência e germinam o mais rápido possivel, quando as condições ambientais são favoráveis (Hopkins \& Graham, 1987).

O método mais utilizado para o controle de plantas daninhas em áreas de pequenos produtores é a capina, realizada manualmente e de acordo com a disponibilidade de mão de obra - geralmente familiar. No ano da coleta, todos os produtores realizaram apenas uma capina manual em seus cultivos. Foi constatado que as plantas daninhas tiveram capacidade de regeneração e consequente produção de sementes, mantendo o banco de sementes, pois não houve cuidados 
mais intensos nos períodos que antecederam as épocas de floração e produção de sementes dessas plantas. Outro fator complicador é que as plantas arrancadas foram deixadas sobre o solo no mesmo local, o que contribui para a incorporação das sementes no solo e, possivelmente, novas infestações.

As espécies de plantas daninhas possuem abundância de sementes armazenadas no solo, as quais podem promover um continuo problema de infestações nas áreas, exigindo manejo adequado para seu controle.

$\mathrm{Na}$ área de estudo, pôde-se perceber um manejo de plantas daninhas extremamente precário, como a utilização do fogo para limpeza da área, que poderá ao longo do tempo tornar o sistema insustentáve1.

Os bancos de sementes das áreas avaliadas foram constituídos predominantemente por espécies daninhas de porte herbáceo, características de áreas agrícolas e ambientes perturbados. No entanto, houve baixa similaridade na composição florística entre os cultivos de mandioca.

Além disso, são necessárias investigações sobre métodos de amostragem no campo para maior precisão da estimação da densidade dos bancos de sementes.

\section{LITERATURA CITADA}

CANOSSA, R. R. et al. Profundidade de semeadura afetando a emergência de plântulas de Alternanthera tenella. Planta Daninha, v. 25, n. 4, p. 719-725, 2007.

CARMONA, R. Bancos de sementes e estabelecimento de plantas daninhas em agroecossistemas. Planta Daninha, v. 13, n. 1, p. 3-9, 1995.

CARMONA, R. Problemática e manejo de bancos de bancos de sementes de invasoras em solos agrícolas.

Planta Daninha, v. 10, n. 1/2, p. 5-16, 1992

CASTRO, P. R. C.; VIEIRA, E. L. Aplicações de reguladores vegetais na agricultura tropical. Guaíba: Agropecuária, 2001. 132 p

CAVERS, P. B. Seed banks: Memory in soil. Can. J. Soil Sci., v. 75, n. 1, p. 11-13, 1995.
CAVERS, P. B.; BENOIT, D. L. Seed banks in arable land. In: LECK, M. A.; PARKER, V. T.; SIMPSON, R. L. (Eds.). Ecology of soil seed banks. New York: Academic Press, 1989. p. $309-328$

CORREIA, N. M. Palhas de sorgo associadas ao herbicida imazamox no controle de plantas daninhas na cultura da soja em sucessão. Planta Daninha, v. 23, n. 3, p. 483-489, 2005.

EPP, G. A. The seed bank of Eupatorium odoratum along successional gradient in a tropical rain forest in Ghana. $\mathbf{J}$. Trop. Ecol., v. 3, n. 2, p. 139-149, 1987.

FERNANDEZ-QUINTANILHA, C. Studying the population dynamics of weeds. Weed Res., v. 28, n. 6, p. $443-447,1988$.

GARCIA-NUNES, C.; AZOCAR, A.; SILVA, J. F. Seed production and soil seed bank in the evergreen woody species from a neotropical savanna. J. Trop. Ecol., v. 17, n. 1, p. $563-576,2003$.

GARWOOD, N. C. Tropical soil seed banks: A review. In: LECK, M. A.; PARKER, V.T.; SIMPSON, R. L. (Eds.) Ecology of soil seed banks. New York: Academic Press, 1989. p. $309-328$.

GORCHOV, D. L. et al. The role of seed dispersal in the natural regeneration of rain forest after strip-cutting in Peruvian Amazon. Vegetation, v. 107/108, p. 339-349, 1993.

HOPKINS, M. S.; GRAHAM, A. W. The species composition of soil seed banks beneath lowland tropical rain forests in north Queensland, Australia. Biotropica, v. 15, n. 2 , p. $90-99,1983$

HOPKINS, M. S.; GRAHAM, A. W. The viability of seeds on rainforest species after experimental soil burials under tropical wet lowland forest in north-eastern Australia. Austr. J. Ecol., v. 12, n. 2, p. 97-108, 1987.

McGEE, A.; FELLER, M. C. Seed banks and disturbed soils in southeastern British Columbia. Can. J. Bot., v. 71, n. 12, p. $1574-1583,1993$

MÜLLER-DUMBOIS, D.; ELLENBERG, H. Aims and methods of vegetation ecology. New York: John Wiley \& Sons, 1974

NOLDIN, J. A. et al. Seed longevity of red rice ecotypes buried in soil. Planta Daninha, v. 24, n. 4, p. 611-620, 2006.

PACHECO, C.; CHAVARRIA, P. L.; MATA, R. H. Herbicidas en el cultivo de la yuca (Manihot esculenta Crantz). Costa Rica: Estación Experimental Fábio Banchit, 1974. 12 p. (Comunicado Técnico, 1) 
PARKER, V. T. et al. Pattern and process in the dynamics of seed banks. In: LECK, M. A.; PARKER, V. T.; SIMPSON, R. L. (Eds.) Ecology of soil seed banks. New York: Academic Press, 1989. p. 25-52.

SOUSA, S. G. A. Dinâmica de plantas invasoras em sistemas agroflorestais implantados em pastagens degradadas na Amazônia Central (Região de ManausAM). 1995. 105 f. Dissertação (Mestrado em Ciências Florestais) - Escola Superior de Agricultura "Luiz de Queiroz”, Universidade de São Paulo, Piracicaba, 1995.

SOUZA, L. S. et al. Efeito alelopático de capim-braquiária (Brachiaria decumbens) sobre o crescimento inicial de sete espécies de plantas cultivadas. Planta Daninha, v. 24, n. 4, p. $657-668,2006$
THOMPSON, K.; GRIME, J.P. Seasonal variation in the seed banks of herbaceous species in ten contrasting habitats. J. Ecol., v. 67, p. 893-921, 1979.

VIDAL, R. A.; TREZZI, M. M. Potencial de utilização de coberturas vegetais de sorgo e milheto na supressão de plantas daninhas em condição de campo: 1- plantas em desenvolvimento vegetativo. Planta Daninha, v. 22, n. 2, p. $217-223,2004$

VISMARA, L. S., OLIVEIRA, V. A.; KARAM, D. Revisão de modelos matemáticos da dinâmica do banco de sementes de plantas daninhas em agrossistemas. Planta Daninha, v. 25, n. 1, p. $1-11,2007$ 